\title{
Models with Memory for RF Power Amplifier Behavioral Modeling
}

\author{
Xiang Chen ${ }^{\mathrm{a}}$, Yihan Xiao ${ }^{\mathrm{b}, *}$, Hui Han ${ }^{\mathrm{a}}$, Jie Chang ${ }^{\mathrm{b}}$, and Nan Tang ${ }^{\mathrm{b}}$ \\ ${ }^{a}$ State Key Laboratory of Complex Electromagnetic Environment Effects on Electronics and Information System (CEMEE), Luoyang, 471003, China \\ ${ }^{b}$ Harbin Engineering University, Harbin, 150001, China
}

\begin{abstract}
Power amplifiers are used to amplify signals to the required power and then transmit the signals through antennas. They are the key component of modern wireless communication systems. However, the power amplifier itself has non-linear characteristics and memory effect, especially when the input signal is a broadband signal and high frequency signal, which seriously affects the normal transmission of communication systems. In this paper, the RF power amplifier under test is an amplifier using a BLT53A transistor. We collect the input and output signals of the power amplifier for behavioral modeling. The behavior model only considers the input and output of the amplifier. We adopt a memory polynomial model based on the least squares method. In this paper, we evaluate the correctness of the model from many aspects including AM-AM curve, AM-PM curve, spectrum, gain compression, constellation diagram, and normalized mean square error. The results show that the model is effective.
\end{abstract}

Keywords: behavioral modeling; power amplifiers (PAs); memory polynomials model

(Submitted on December 25, 2018; Revised on January 23, 2019; Accepted on February 20, 2019)

(C) 2019 Totem Publisher, Inc. All rights reserved.

\section{Introduction}

The frequency source and power amplifier are the most important nonlinear devices of the radiation source, and they are the main source of fingerprint characteristics of the individual. With the continuous development of microelectronics technology, the phase noise, harmonics, and spectral spurs of the frequency source are further improved. It is increasingly difficult to extract individual subtle features from the related spurious characteristics parameters caused by the frequency source. The internal complex structure of the power amplifier is designed and manufactured to cover various processes such as DC circuit, RF circuit, control circuit, protection circuit, and shield circuit design, and the RF circuit design also includes various processes such as the selection of crystal amplifier and matching network design. Even if the amplifier is fabricated according to the same specifications, it is impossible to obtain an RF power amplifier with the same parameters. Due to inevitable nonlinear characteristics such as intermodulation interference and intermodulation interference of power amplifiers, some power amplifiers must work in high power and non-linear states, and they will exhibit distinct individual characteristics. Power amplifiers have become an excellent object for studying the mechanism of the generation of fine features of radiation sources [1].

To deeply study the mechanism of generating fine features of power amplifiers, it is necessary to start from the design of power amplifiers, including power amplifier index design, circuit schematic simulation, PCB layout design, and physical processing. This gives us clear control over the power amplifier from simulation to physical production, providing us with a transparent and anatomical object. Therefore, we can accurately locate the various influencing factors and even circuit components in the power amplifier circuit in the later analysis of the mechanism of fine feature generation.

The extracted subtle features should be distinguished from ordinary modulation recognition, and the input signal excitation effects such as modulation factors are small. To do this, it is necessary to establish a behavioral model of the amplifier, use the input and output data to characterize the amplifier, and ensure that the modeled amplifier object is used for fine feature extraction and the system characteristics remain unchanged in order to focus on studying the influence of the

\footnotetext{
* Corresponding author.

E-mail address: xiaoyihan@hrbeu.edu.cn
} 
input signal to find a more stable feature.

According to whether the behavioral model contains memory effect, it can be classified into two categories: memoryless models and models with memory. Whether there is a memory effect in the power amplifier depends on whether the output signal is related to the historical input signal. The earliest study of the modeling of power amplifiers is a memoryless model. The earliest study of the modeling of power amplifiers is a model with no memory effect. A commonly used memoryless model involves using the power series of the input signal to represent the output signal of the amplifier, mainly including orthogonal power series and complex coefficient power series. Another commonly used memoryless model is the Saleh model named after A.A.M. Saleh in 1981. This model can be used for traveling wave tube amplifiers (TWTA), but the model is based on monophonic sounds and does not reflect the distortion of various harmonics. After that, the memory effect is gradually recognized by people. However, in narrowband applications, since the bandwidth of the signal is less than the bandwidth of the device, the memory effect has less of an effect on the amplifier [2]. Now, with the popularization of third generation communication technology and the rise of technologies used in the third generation communication system such as WCDMA and CDMA2000, the memory effect of the power amplifier has become more apparent due to the widening of the signal bandwidth [3]. Therefore, constructing a model with memory effect becomes more important for the design of power amplifiers and system optimization. Many works have been carried out on the establishment of the memory effect model. In 1989, Bosch and Gatti proposed a method for measuring and simulating the memory effect of a power amplifier and proved that there is a memory effect in the power amplifier [4]. Vuolevi and Rahkonen et al. defined the memory effect of the amplifier and gave the reasons for the memory effect [5]. Boumaiza and Ghannouchi analyzed the thermal memory effects of the power amplifier and established a model with memory related to thermal changes [6]. Liu studied the standard of electrical memory effects in broadband power amplifiers [7]. So far, there are many models with memory, each with different characteristics.

For behavioral modeling, artificial neural networks are effective methods. Artificial neural networks do not need to understand the details of the circuit. It can train the neural network well and obtain a good behavioral model only by using the input and output data of the circuit. A recurrent neural network is a neural network that can learn the dynamic behavior of a system. This neural network is used in many fields, such as speech recognition [8-9], system identification [10-12], and signal processing and control [13]. Recurrent neural networks differ from other neural networks in that their inputs and outputs contain time delay information, and the neural network is trained with time domain data, which can better reflect the behavior of the nonlinear power amplifier.

In this paper, we focus on the nonlinear behavior of the power amplifier such as the power gain transfer function, gain compression (AM-AM), and transfer phase (AM-PM) characteristics. This behavior occurs at the envelope of the signal.

\section{Basic Theory}

Least squares arises from data fitting problems. It is a method for estimating parameters in a mathematical model based on the sum of the squares of the differences between the observed data and the model data. The approximate functional relationship between the input data $t$ and the output data $y$ is as follows:

$$
y=\phi(x, t)
$$

Where $x \in R^{n}$ is the pending parameter.

In order to estimate the value of the parameter $x$, it is necessary to obtain the observation data $\left(t_{1}, y_{1}\right),\left(t_{1}, y_{1}\right), \cdots,\left(t_{1}, y_{1}\right)$ after several experiments and then calculate the squared error of the error between the model output value and the actual observation value, which is

$$
\sum_{i=1}^{m}\left(y_{i}-\phi\left(\begin{array}{lll}
x & t_{i} & 3
\end{array}\right)\right.
$$

Minimize the sum of the squares of the errors to determine the estimate of the parameter $x$, that is, use the method of least squares. Generally speaking, the establishment of this method must satisfy the premise of $m<n$.

If we introduce a function 


$$
r_{i}=y_{i}-\phi(x, t), \dot{E} 1,2 \cdot, 3
$$

And make

$$
r(x)=\left(r_{1}(x), r_{2}(x), \cdots, r_{n}(x)\right)
$$

Then, the least squares problem is equivalent to

$$
\min _{\mathrm{x} \in R^{n}} r(x)^{\mathrm{T}} r(x)
$$

If the model function estimation in the least squares problem is accurate, the merit of the least squares problem is very close to zero. Therefore, $r(x)$ is often called the residual function.

For the linear least squares problem, the residual function can be represented as follows:

$$
r(x)=b-A x
$$

Thus, the linear least squares problem can be expressed as

$$
\min _{\mathrm{x} \in R^{n}}\|b-A x\|^{2}
$$

If $A$ is full rank, considering that the stable point of the quadratic convex function is a small value point, we can directly get the solution formula of $x$, which is

$$
x=\left(A^{\mathrm{T}} A\right)^{-1} A \bar{b}
$$

For the complex domain,

$$
\min _{\mathrm{x} \in C^{n}}\|b-A x\|^{2}
$$

We can also directly obtain the solution formula of $x$, which is

$$
x=\left(\bar{A}^{\mathrm{T}} A\right)^{-1} \bar{A}^{\mathrm{T}} b
$$

\section{Modeling Approaches}

\subsection{Behavioral Model Structure}

Behavior modeling of power amplifiers refers to calculating a functional relationship or a model to adapt to the non-linear characteristics of power amplifiers. Behavior modeling is a kind of modeling method that regards the power amplifier as a black box. This method establishes the input and output (I/O) measurement model of power amplifiers and adopts the theory of nonlinear system optimization. Behavior modeling extracts a set of functions and their parameters. Compared with circuit layer modeling, behavior modeling neglects the specific circuit form of power amplifiers and only focuses on the input and output of power amplifiers. We obtain prior knowledge of nonlinearity from power amplifiers. Generally speaking, this nonlinearity uses a prior input-output characteristic of the power amplifier, and the behavior modeling problem is transformed into calculating function parameters by using the least squares function parameter estimation method. In this paper, we use the least squares method to modeling the behavior of the power amplifier [14]. Figure 1 shows the method flow of behavioral modeling for power amplifiers.

AM-AM and AM-PM characteristics are always measured in modeling. Simple monotone measurements are considered in the initial stage. Narrow-band nonlinear amplifier is used to model. Then, the polynomial is fitted to the measured data, and a model is obtained, which can be used to predict the non-linear distortion in the communication system through computer simulation. However, this simple model based on monotone measurement cannot be used to simulate the 
broadband behavior of nonlinear amplifiers, because the frequency dependence of monotone characteristics makes the nonlinear amplifiers have memory effect.

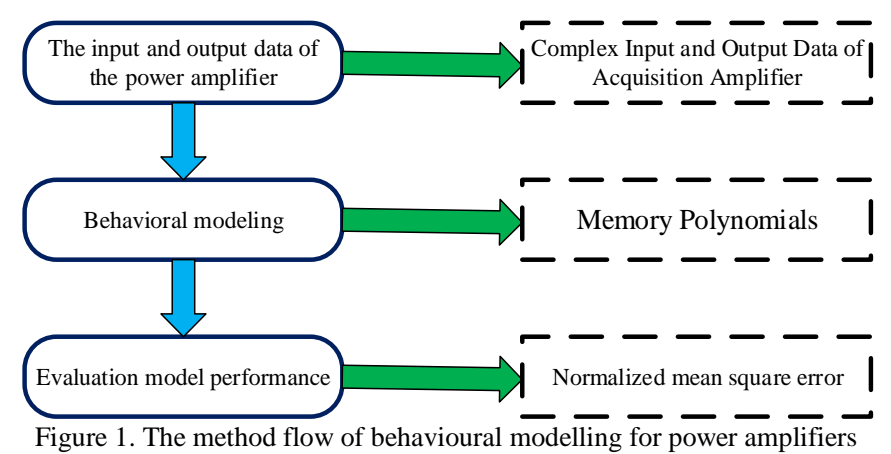

\subsection{General Volterra Series Model}

Most of the nonlinear analysis models are based on Volterra series analysis and Wiener's nonlinear memory system modeling theory. In general Volterra series analysis, the nonlinear model usually consists of linear or nonlinear filters with limited bandwidth, which determine the frequency selectivity of the system. In the time domain, these filters are represented by their kernel functions (impulse response), where the support of the kernel represents memory in the system. Many variants of the generic Volterra model, including the memory-free system model, can be defined by defining the Volterra kernel relationship, as follows [14].

A general Volterra series model of a nonlinear system is described by the following functional expression of continuity:

$$
y(t)=\sum_{n=1}^{\infty} F_{n}(x(t))=\sum_{n=0}^{\infty} y_{n}(t)
$$

Where $F_{n}(x(t))$ is the Volterra function. It is defined as

$$
F_{n}\left(x(y)=\int_{-\infty}^{\infty} \cdots \int_{-\infty}^{\infty} \not\left(k ; \lambda_{1} ; \cdots \lambda\right) \prod_{i=1}^{n}(x)_{i} \mathrm{~d}\right.
$$

Where $h_{n}\left(\lambda_{1}, \cdots, \lambda_{n}\right)$ is the n-dimensional Volterra kernel that can be symmetric without loss of generality. Volterra kernel support defines the memory characteristics of power amplifiers. It can be seen from the formula that the current output of the amplifier is affected not only by the current input, but also by the previous input. We call this phenomenon the memory effect. Figure 2 shows a block diagram of the Volterra model. General Volterra series analysis is an analytical method for nonlinear modeling, because it represents nonlinearity in a similar way as Taylor series pair analysis function. Therefore, a Volterra series can be described as a Taylor series with memory. The convergence of the sequence can be guaranteed only in the limited range of input amplitude (or power). Generally speaking, for any continuous nonlinear system approximated by Volterra series expansion, the input signal must constitute a subset of the input function space. In practice, the series approximation holds only on finite time intervals where the input signal is nonzero [14].

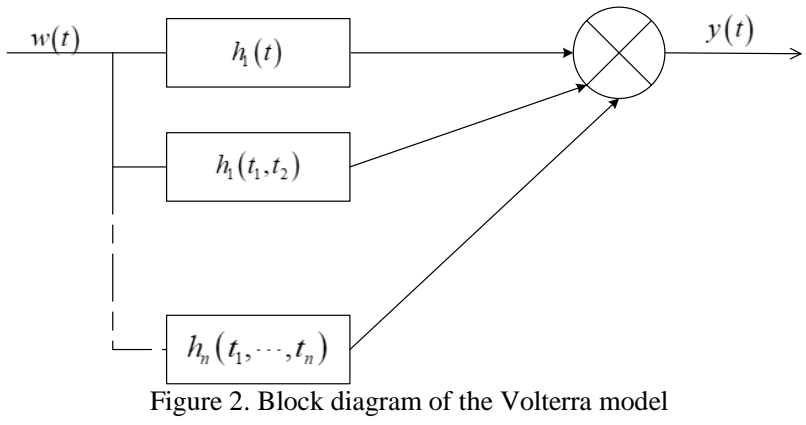

Next, we will discuss variants of the generic Volterra model, which makes the development of system parameters easier 
to handle by simplifying the kernel relationships. However, for all these models, the kernel relationship must remain unchanged. In some cases, this can be verified by an intuitive understanding of the system.

\subsection{Memory Polynomials}

A memory polynomial is a special case of the general Volterra model in discrete time. It is widely used in various applications in literature, especially in the linearization of power amplifiers with memory. To develop memory polynomials, we will consider a general Volterra model with finite memory, which can be expressed in discrete time:

$$
y(n)=\sum_{k=1}^{N} y_{k}(n)
$$

Where $y_{k}(n)$ is the $k$-dimensional convolution of the input with the kth-order Volterra kernel:

$$
y_{k}(n)=\sum_{m_{1}=0}^{P-1} \cdots \sum_{m_{k}=0}^{P-1} h_{k}\left(m_{1}, \cdots, m_{k}\right) \prod_{l=1}^{k} x\left(n-m_{l}\right)
$$

Which represents a generalization of a power series representation with a finite memory of length $P$. By making a change of variables to the diagonal index variables:

$$
n_{l}=m_{l+1}-m_{1}, l=1,2, \cdots, k-1
$$

Thus, the generalized Hammerstein model can be formulated as:

$$
y(n)=\sum_{K=1}^{N} \sum_{m=0}^{P-1} a_{k m} \tilde{x}(n-m)|x(n-m)|^{k}
$$

A narrowband form of the Hammerstein model can be obtained by taking the components of the nonlinear output that lie on the fundamental frequency. Thus, only combinations of the form $x(n)|x(n-m)|^{k-1}$ are considered, and hence the complex envelope of the output can be written in terms of the complex envelope of the input in the form of a memory polynomial of the form [14]:

$$
\tilde{y}(n)=\sum_{K=1}^{N} \sum_{m=0}^{P-1} a_{k m} \tilde{x}(n-m)|\tilde{x}(n-m)|^{k}
$$

\section{Behavioral Modeling based on Real Data of Power Amplifier}

\subsection{Measurements and Simulation for Model Generation}

The RF power amplifier under test is an amplifier using a BLT53A transistor. The amplifier can be used for digital signal transmission, interphone, aero mode remote control, and meter reading systems. It is a power amplifier based on silicon technology, providing wide bandwidth operation support from DC to 3G. It has very high efficiency. The power efficiency can reach $65 \%$ when the power supply is 6 volts and the output is $36 \mathrm{dBm}$ at $433 \mathrm{MHz}$ operating frequency. It has an antistatic interference protection diode inside, which can resist ESD very well and avoid damage to the device. BLT53A is very suitable for some data transmission systems, and in some specific occasions it may require 2 watts of communication power. An illustration of the fixture is shown in Figure 3.

The data acquisition method and the experimental process are shown in Figure 4. Generate signals from PC at MATLAB and export fixed signal format for the signal generator. The signal generated by MATLAB is the baseband signal. The signal generator is used to set up carrier frequency up conversion to transmit the signal. The signal generator can set the carrier frequency and input power of the input signal. MATLAB can produce a variety of modulation signal styles. The maximum power output from the signal generator to the power amplifier is $10 \mathrm{dBm}$. During the acquisition process, the signal generator is used to change the input power, modulation mode, and center frequency of the input signal of the power amplifier. We use a complex digitally modulated baseband signal for the behavioral modeling of the power amplifier. The 
modulation mode of the signal is 16QAM. The baseband signal data is generated by MATLAB in PC. After the signal is generated, the signal is input to the amplifier by the signal generator.

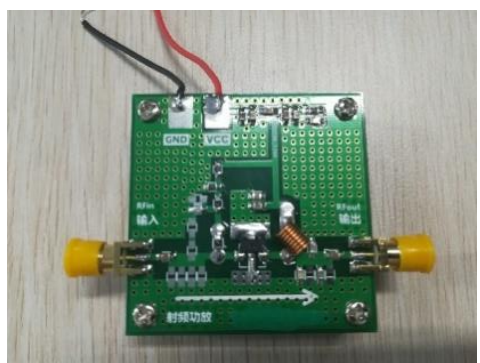

Figure 3. The BLT53A

The test voltage of the amplifier is 5V. A DC regulated power supply can provide stable voltage. Ensure that the voltage of the amplifier is stable rather than variable at work. We need input data and output data of the power amplifier for modeling. Therefore, we need a power divider to divide the output signal of the signal generator into two identical signals. One of them is directly collected by one channel of the oscilloscope. Another signal serves as input to the power amplifier. The modulation style of the input signal is 16QAM. The power input is $10 \mathrm{dBm}$, and the carrier frequency is $433 \mathrm{MHz}$. The bandwidth of the signal is $1 \mathrm{MHz}$. To protect the oscilloscope from damage due to high power signal input, an attenuator is connected between the amplifier output and the oscilloscope acquisition channel. We collect the output signal of the power amplifier by one channel of the oscilloscope. The sample rate is $10 \mathrm{MSa} / \mathrm{s}$. According to the Nyquist sampling theorem, $10 \mathrm{MSa} / \mathrm{s}$ is enough to ensure signal integrity. Through the above steps, we can obtain the input and output signal data for behavioral modeling.

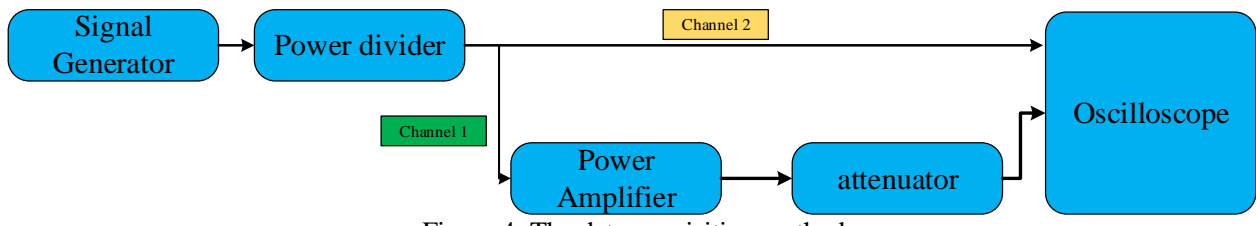

Figure 4. The data acquisition method

\subsection{Memory Polynomials Model Extraction}

Table 1 shows the algorithm flow of the memory polynomial model used in this paper.

Table 1. Memory polynomials model extraction

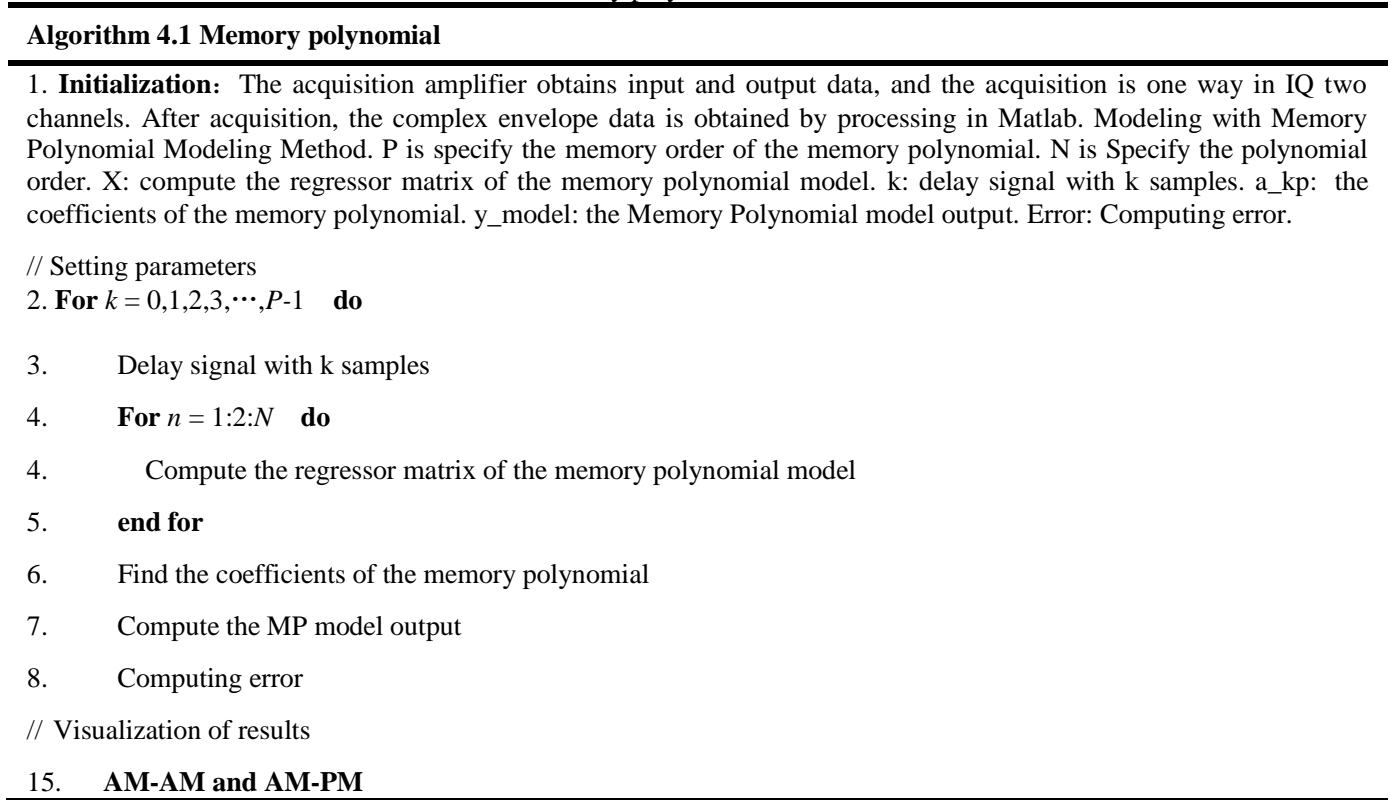




\subsection{Performance Analysis}

Firstly, the data is received and collected, and a signal sample of input and output is intercepted as the research object. Its input and output waveforms are shown in Figure 5. Because of the sampling error of the receiving device, the input and output will have a certain time deviation in the time domain. The input and output are aligned by correlation, and the waveform is shown in Figure 6. After aligning the data, the data is down-converted and the input and output data after down-converting is modeled. After obtaining the modeled data, the basic analysis is carried out.

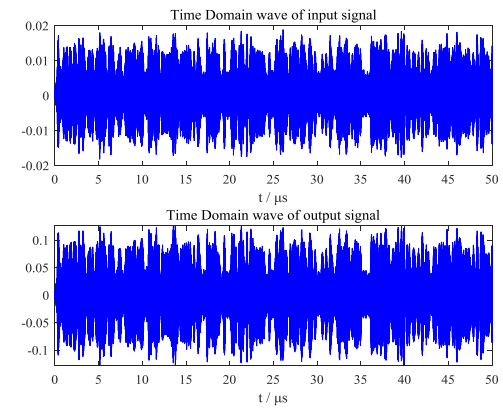

Figure 5. The input and output waveforms

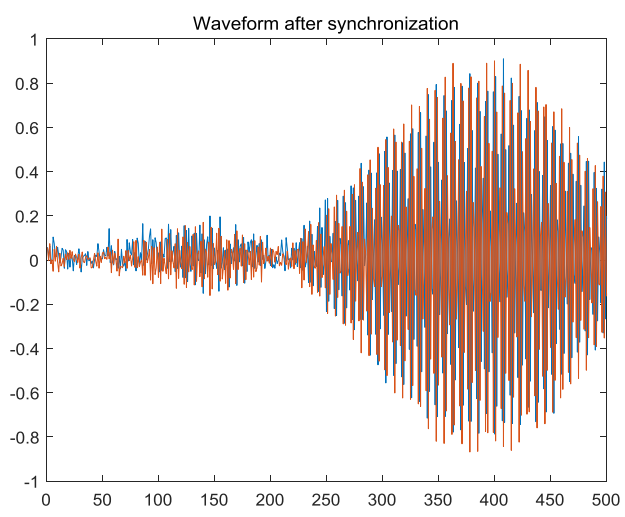

Figure 6. The input and output waveforms after time synchronization

Next, we use the method of memory polynomial to model its behavior. The order of polynomial is 3 , and the memory depth is 3. Figure 7 shows comparisons between the model results and original test data after memory polynomial modeling. Firstly, we use the AM-AM characteristic curve and AM-PM characteristic curve to evaluate the modeling performance of the model. In the AM-AM curve, abscissa is the amplitude data of the input signal and ordinate is the output amplitude of the output signal. It can be seen from the figure that divergence and detention occur in the AM-AM characteristic curve and AM-PM curve, which are the characterization of the non-linear characteristics of the amplifier. In the figure, the blue dots represent the fitting effect of the model.
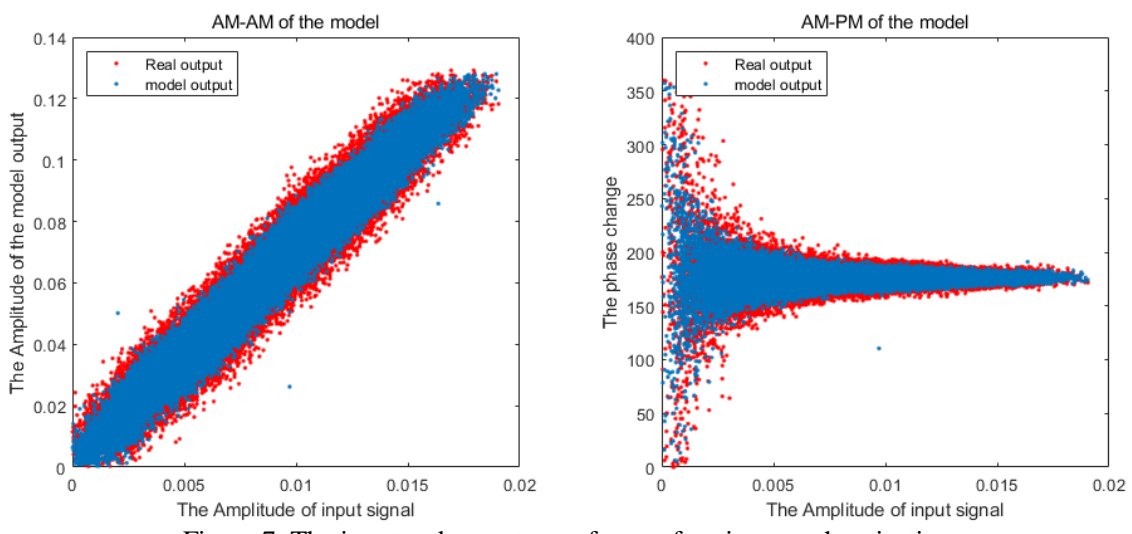

Figure 7. The input and output waveforms after time synchronization 
Figure 8 shows the spectrum of the real output data and the model output data. The result shows that the model can fit the data in frequency domain. Figure 9 shows the gain compression of the real data and the model data. The result shows that the model can fit the behavior of the power amplifier by gain compression. Figure 10 shows the constellation diagram of the real data and the model data. These results show that the model can fit the behavior of the power amplifier in several aspects and the method is effective.
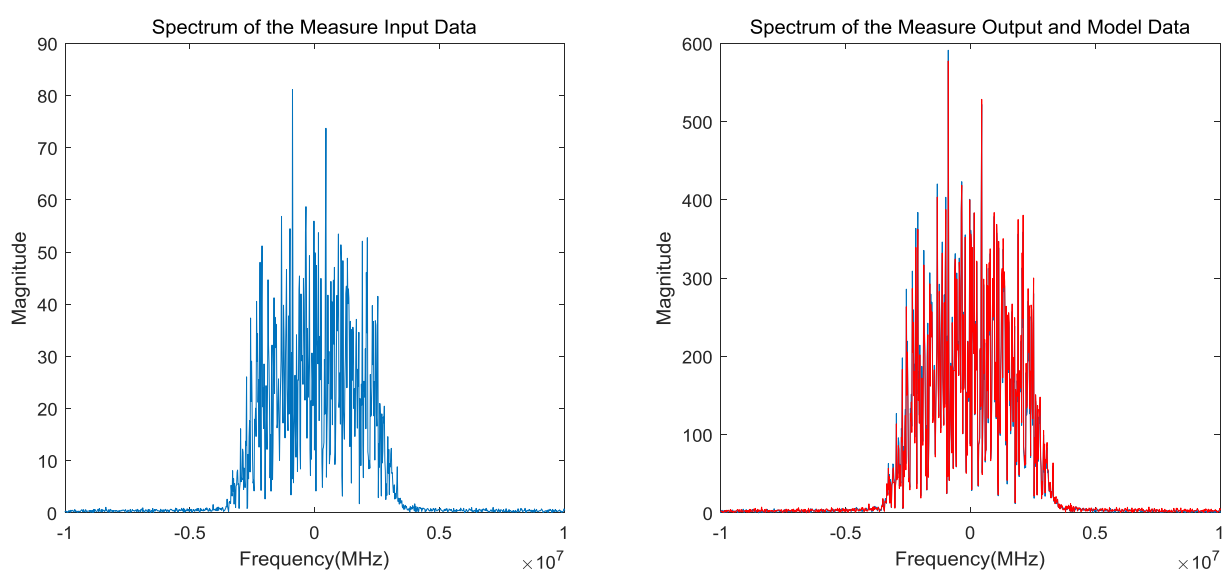

Figure 8. The spectrum of the measure data and model data

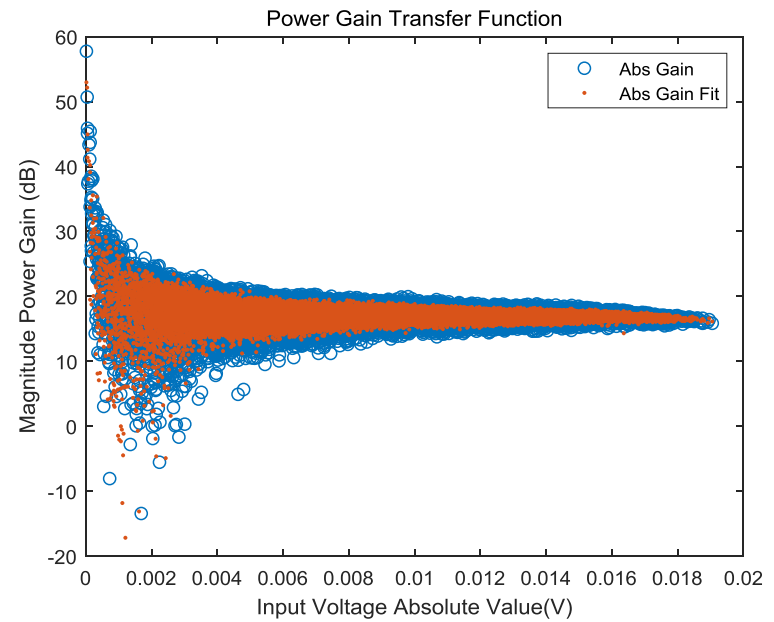

Figure 9. The spectrum of the measure data and model data
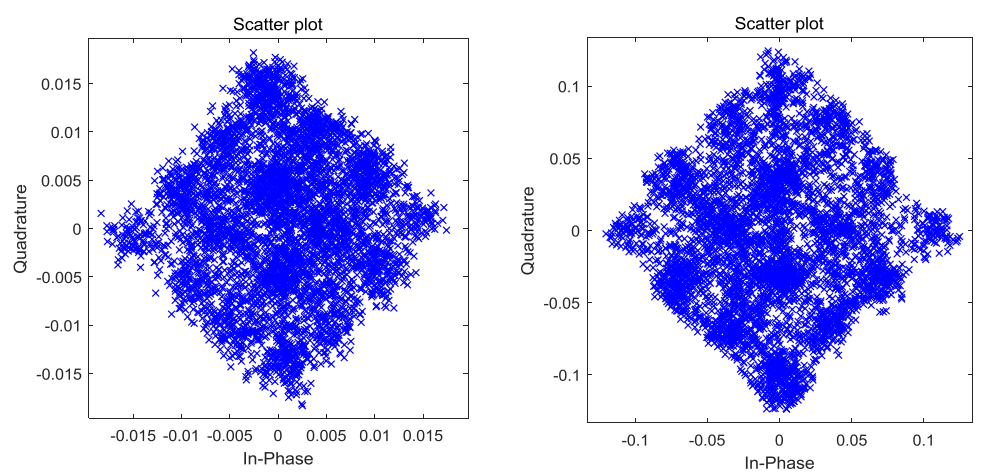

Figure 10. The spectrum of the measure data and model data

Table 2 shows the NMSE with different depth of memory and polynomial order. The results show that the accuracy of the model increases with the increase in the order of the model. 
Table 2. The NMSE with different depth of memory and polynomial order

\begin{tabular}{c|c|c|c|c|c|c|c}
\hline NMSE & $P=1$ & $P=2$ & $P=3$ & $P=4$ & $P=5$ & $P=6$ & $P=7$ \\
\hline$N=1$ & $\mathbf{- 1 7 . 2 5 7 3}$ & -20.0010 & -20.6399 & -20.8332 & -21.4167 & -21.7474 & -21.9383 \\
\hline$N=2$ & & -20.0010 & & -20.8332 & & -21.7474 & \\
\hline$N=3$ & -17.2769 & -20.0064 & -20.6449 & -20.8380 & -21.4213 & -21.7524 & -21.9439 \\
\hline$N=4$ & & -20.0064 & & -20.8380 & & -21.7524 & \\
\hline$N=5$ & -17.2808 & -20.0080 & -20.6457 & -20.8390 & -21.4226 & -21.7537 & -21.9452 \\
\hline$N=6$ & & -20.0080 & & -20.8390 & & -21.7537 & \\
\hline$N=7$ & -17.2808 & -20.0080 & -20.6457 & -20.8390 & -21.4226 & -21.7537 & $\mathbf{- 2 1 . 9 4 5 2}$ \\
\hline
\end{tabular}

\section{Conclusions}

In this paper, the RF power amplifier under test is an amplifier using a BLT53A transistor. We collect the input and output signals of the power amplifier for behavioral modeling. We collect the output signal of the power amplifier by one channel of the oscilloscope. We adopt a memory polynomial model based on the least squares method. In this paper, we evaluate the correctness of the model from many aspects including AM-AM curve, AM-PM curve, spectrum, gain compression, constellation diagram, and normalized mean square error. By observing the time domain waveform, AM-AM curve, AMPM curve, gain compression, and constellation of the output signal of the model, the results show that the model can match the behavior of the power amplifier from multiple aspects. The NMSE also can evaluate the accuracy of the model. The smaller the value of the NMSE, the higher the accuracy. The order of the model influences the performance of the model. In future works, we hope to establish a more accurate model to model the amplifier.

\section{Acknowledgments}

This work is supported by the National Natural Science Foundation of China (No. 61771154), the Fundamental Research Funds for the Central Universities (No. HEUCFG201830), and the State Key Laboratory of CEMEE (No. CEMEE2018K0104A).

\section{References}

1. S. Cripps, "RF Power Amplifiers for Wireless Communications," IEEE Microwave Magazine, Vol. 1, No. 1, pp. 64, Artech House, Boston, 2006

2. A. A. M. Saleh, "Frequency-Dependent and Frequency-Independent Nonlinear Models of TWT Amplifiers," IEEE Transactions on Communications, Vol. COM-29, No. 11, pp. 1715-1720, November 1981

3. L. Ding, Z. X. Ma, D. R. Morgan, M. Zierdt, and J. Pastalan, "A Least-Squares/Newton Method for Digital Predistortion of Wideband Signals," IEEE Transactions on Communications, Vol. 54, No. 5, pp. 833-840, 2006

4. W. Bosch and G. Gatti, "Measurement and Simulation of Memory Effects in Predistortion Linearizer," IEEE Transactions on Microwave Theory and Techniques., Vol. 37, No. 12, pp. 1885-1890, 1989

5. J. H. K. Vuolevi, T. Rahkonen, and J. P. A. Manninen, "Measurement Technique for Characterizing Memory Effects in RF Power Amplifiers," IEEE Transactions on Microwave Theory and Technology, Vol. 49, No. 8, pp. 1383-1388, 2001

6. S. Boumaiza and F. M. Ghannouchi, "Thermal Memory Effects Modeling and Compensation in RF Power Amplifiers and Predistortion Linearizers," IEEE Transactions on Microwave Theory \& Technology, Vol. 51, No. 12, pp. 2427-2433, 2003

7. T. J. Liu, S. Boumaiza, A. B. Sesay, and F. M. Ghannouchi, "Quantitative Measurements of Memory Effects in Wideband RF Power Amplifiers Driven by Modulated Signals," IEEE Microwave and Wireless Components Letters, Vol. 17, No. 1, pp. 7981,2007

8. C. Jeyalakshmi, V. Krishnamurthi, and A. Revathi, "Speech Recognition of Deaf and Hard of Hearing People using Hybrid Neural Network," in Proceedings of Mechanical and Electronics Engineering 2nd International Conference, Vol. 1, No. V1-83V1-87, August 2010

9. B. Lu, J. J. Wu, Y. Wang, and J. P. Li, "A Speech Recognition System based on Multiple Neural Networks," in Proceedings of Sixth International Conference on Natural Computation, Vol. 1, pp. 48-51, August 2010

10. Z. G. Wang and X. Zhang, "Using Neural Network Realize the Identification of Nonlinear System," in Proceedings of 2011 International Conference on Electric Information and Control Engineering, pp. 3777-3779, April 2011

11. W. J. Zhang, Z. J. Liu, J. S. Zhu, and X. K. Xu, "Identification and Control of Time-Delay System with Recurrent Wavelet Neural Networks," in Proceedings of Third International Conference on Intelligent Control and Information Processing, pp. 211-216, July 2012

12. Y. Shen, X. L. Ju, and C. X. Liu, "Application of Second Order Diagonal Recurrent Neural Network in Nonlinear System Identification," Web Information Systems and Mining, Vol. 1, pp. 420-424, October 2010

13. H. Drucker, C. J. Burges, L. Kaufman, A. Smola, and V. Vapnik, "Support Vector Regression Machines," Advances in Neural Information Processing Systems, Vol. 9, pp. 155-161, 1997

14. M. G. Khaled, "Simulation of Nonlinear Systems in Simulink®," Nonlinear Distortion in Wireless Systems: Modeling and Simulation with MATLAB, IEEE, 2012 